\title{
Tools and Apps to Enhance Situational Awareness for Global Disease Surveillance
}

\author{
Alina Deshpande*, Kristen Margevicius, Eric Generous, Kirsten Taylor-McCabe, Lauren \\ Castro, Joseph Longo and Reid Priedhorsky
}

Defense Systems and Analysis Division, Bioscience Division, Los Alamos National Laboratory, Los Alamos, NM, USA

\section{Objective}

To develop a suite of tools that provides actionable information and knowledge for enhanced situational awareness during an unfolding event such as an infectious disease outbreak.

\section{Introduction}

Situational awareness is important for both early warning and early detection of a disease outbreak, and analytics and tools that furnish information on how an infectious outbreak would either emerge or unfold provide enhanced situational awareness for decision makers/ analysts/public health officials, and support planning for prevention or mitigation. Data sharing and expert analysis of incoming information are key to enhancing situational awareness of an unfolding event. In this presentation, we will describe a suite of tools developed at Los Alamos National Laboratory (LANL) that provide actionable information and knowledge for enhanced situational awareness during an unfolding event; The biosurveillance resource directory (BRD), the biosurveillance analytics resource directory (BaRD) and the surveillance window app (SWAP).

\section{Methods}

The BRD is a database that provides a one-stop shop for disease surveillance resources and can be used for validating information received about disease outbreaks. The BRD is searchable by multiple keywords (e.g. location, data stream use, sponsor, disease). Disease prediction, forecasting and monitoring models are useful for disease management. However, for a given disease outbreak, without knowledge of their context it is very difficult to determine what epidemiological model could be used or whether a particular model is available for use. Moreover, given the diversity of model types and the manner in which they are described use of the appropriate model is also a challenge. LANL is developing a reference tool, the BaRD that enables rapid selection of appropriate models for use in disease prediction, monitoring or forecasting. The BaRD catalogs and classifies model specific information and can be used to find the appropriate epidemiological model to use if it was available for a specific disease.

Data obtained in isolation has little value to a decision maker. A local public health official who receives a report about a few cases of gastrointestinal illness in his/her county may be able to plan a more effective infection control strategy if they had knowledge about what the typical disease outbreak curve looks like, the duration of time it takes to spread through a population, how many cases are typical for that region of the world - is it too late to control the infection or do they have time to act? They need a frame of reference and context in order to determine decisions to be made. The SWAP is being developed to provide a more robust understanding of a rapidly unfolding event through graphical visualization. It is intended to provide a frame of reference for incoming data for a particular location and time during a disease outbreak, so that the user is able to understand and qualify the situation to facilitate actions to prevent, stop or mitigate the event.

\section{Results}

The BRD has been pilot tested and is transitioning to a web hosted version that will be accessible to the global disease surveillance community. It currently contains information on over 296 resources for infectious disease surveillance, representing U.S. and international government agencies, non-profits, commercial and industry businesses, and academic institutions. A robust and comprehensive framework that describes the fundamental elements of infectious disease surveillance underlies this tool. The BaRD and SWAP are tools that are currently under development and are also intended to be available to the global disease surveillance community through web versions. A systematic model characterization framework has been developed to allow epidemiological model description and "apples to apples" comparison for multiple models available for the same disease. The SWAP is based on detailed analyses of historical disease specific outbreaks (e.g. cholera) around the globe.

\section{Conclusions}

The suite of tools being developed at LANL offer robust decision support and provide actionable information that facilitates situational awareness in infectious disease surveillance. The tools will be evaluated for operational utility and ease of use once fully mature and web hosted.

\section{Keywords}

infectious disease; decision support; surveillance

\section{Acknowledgments}

We would like to acknowledge The Defense Threat Reduction Agency (DTRA) - Joint Science and Technology Office (JSTO) for sponsoring this project.

\author{
*Alina Deshpande \\ E-mail: deshpande_a@lanl.gov
}

